\title{
Theory of Phase Reconstructed Vector Field Electron Tomography
}

\author{
M. De Graef* and M. Beleggia** \\ * Department of Materials Science \& Engineering, Carnegie Mellon University, Pittsburgh, PA 15213 \\ ** Materials Science Division, Brookhaven National Laboratory, Upton, NY 11973
}

In this contribution, we describe a method to reconstruct the 3D magnetic induction inside and around a magnetized particle, and we present results of numerical simulations indicating the degree of accuracy of this type of reconstruction. The phase of an electron wave traveling through a region of space with an electrostatic potential $V(\mathbf{r})$ and a magnetic vector potential $\mathbf{A}(\mathbf{r})$ is given by:

$$
\varphi\left(\mathbf{r}_{\perp}\right)=\varphi_{e}\left(\mathbf{r}_{\perp}\right)+\varphi_{m}\left(\mathbf{r}_{\perp}\right)=C_{E} \int_{-\infty}^{+\infty} V\left(\mathbf{r}_{\perp}+\ell \hat{\boldsymbol{\omega}}\right) \mathrm{d} \ell-C_{B} \int_{-\infty}^{+\infty} \mathbf{A}\left(\mathbf{r}_{\perp}+\ell \hat{\boldsymbol{\omega}}\right) \cdot \hat{\boldsymbol{\omega}} \mathrm{d} \ell=C_{E} \mathcal{P}_{\hat{\boldsymbol{\omega}}}-C_{B} \mathcal{V}_{\hat{\boldsymbol{\omega}}}
$$

The constants are $C_{E}=\frac{\pi}{E \lambda}$, with $E$ the relativistic electron accelerating potential and $\lambda$ the wavelength; $C_{B}=\frac{e}{\hbar}$. The integration, parametrized by $\ell$, occurs along the projection direction with unit direction vector $\hat{\boldsymbol{\omega}}$. The position vector $\mathbf{r}_{\perp}$ lies in the projection plane, so that $\hat{\boldsymbol{\omega}} \cdot \mathbf{r}_{\perp}=0$. The integrals above are known as the $3 \mathrm{D}$ scalar $X$-ray transform, $\mathcal{P}_{\hat{\boldsymbol{\omega}}}$, and the vectorial $X$-ray transform, $\mathcal{V}_{\hat{\boldsymbol{\omega}}}$.

In order to reconstruct both $V$ and $\mathbf{A}$ (or rather, $\nabla \times \mathbf{A}=\mathbf{B}$ ), we must first separate the electrostatic phase from the magnetic phase, since the slice theorems for scalar and vector quantities are different. Flipping the sample by $180^{\circ}$ (i.e., projecting along the direction $-\hat{\boldsymbol{\omega}}$ ) and recording a second phase map allows for the separation of the two phase shifts:

$$
\left(\mathcal{P}_{\hat{\boldsymbol{\omega}}} V\right)\left(\mathbf{r}_{\perp}\right)=\frac{1}{2 C_{E}}\left[\varphi_{\hat{\boldsymbol{\omega}}}\left(\mathbf{r}_{\perp}\right)+\varphi_{-\hat{\boldsymbol{\omega}}}\left(\mathbf{r}_{\perp}\right)\right] ; \quad\left(\mathcal{V}_{\hat{\boldsymbol{\omega}}} \mathbf{A}\right)\left(\mathbf{r}_{\perp}\right)=\frac{1}{2 C_{B}}\left[\varphi_{\hat{\boldsymbol{\omega}}}\left(\mathbf{r}_{\perp}\right)-\varphi_{-\hat{\boldsymbol{\omega}}}\left(\mathbf{r}_{\perp}\right)\right] .
$$

It can be shown (e.g. [1]) that there is an analog to the Fourier slice theorem for vector fields:

$$
\mathrm{i} \kappa\left(\tilde{\mathcal{V}}_{\hat{\boldsymbol{\omega}}} \mathbf{A}\right)(\kappa)=-\tilde{B}(\kappa \cos \theta, \kappa \sin \theta),
$$

where $(\kappa, \theta)$ are polar coordinates in a 2D Fourier plane, a tilde indicates a Fourier transformed quantity and $B$ is the induction component normal to the tilt plane. This suggests the following procedure: Obtain 2 tilt series around two orthogonal tilt axes, covering as large an angular range as possible. Then flip the sample over and obtain another 2 tilt series, for the same orientation of the tilt axes (in other words, for the orientations $\hat{\omega}$ and $-\hat{\omega}$ ). The "images" of each tilt series would be either holograms (from which the phase can be extracted), or three-member through-focus series (when the Transport-of-Intensity formalism is used to extract the phase). All phase images must then be aligned, first in $(\hat{\boldsymbol{\omega}},-\hat{\boldsymbol{\omega}})$ pairs, so that the magnetic phase shift can be extract using eq. (2). Then, the magnetic phase shifts must be aligned with respect to each other for the tomographic reconstruction.

If we select the first column of pixels from the first tilt series (tilt angles $\alpha_{j}$ ), then we have a series of projections of the electrostatic and vector potential from which we can reconstruct 2D slices:

$$
\left(\mathcal{P}_{\hat{\boldsymbol{\omega}}} V\right)_{m \alpha_{j}}\left(k_{y}\right)=\tilde{V}\left(k_{y} \hat{\mathbf{n}}_{j}\right) ; \quad i k_{y}\left(\mathcal{V}_{\hat{\boldsymbol{\omega}}} \mathbf{A}\right)_{m \alpha_{j}}\left(k_{y}\right)=-\tilde{B}_{x}\left(k_{y} \hat{\mathbf{n}}_{j}\right) .
$$

Repeating this for each column $m$, we obtain the 3D electrostatic potential, and the 3D component $B_{x}(\mathbf{r})$ of the magnetic induction. For the tilts around the $\mathbf{e}_{y}$ axis (tilt angles $\beta_{j}$ ), we apply

$$
i k_{x}\left(\mathcal{V}_{\hat{\boldsymbol{\omega}}} \mathbf{A}\right)_{\beta_{j} n}\left(k_{x}\right)=-\tilde{B}_{y}\left(k_{x} \hat{\mathbf{n}}_{j}\right),
$$


so that we have both the $x$ and $y$ components of the magnetic induction. Finally, we apply the condition $\nabla \cdot \mathbf{B}=0$ to determine the $z$-component of $\mathbf{B}$.

Consider a uniformly magnetized sphere with radius $R=32 \mathrm{~nm}$, saturation induction $B_{0}=1 \mathrm{~T}$, and magnetization direction $\hat{\mathbf{m}}=\left[\cos \frac{\pi}{6}, \sin \frac{\pi}{6}, 0\right]$. The phase shift is given by [2] $\left(j_{1}(x)\right.$ is the spherical Bessel function of the first type):

$$
\tilde{\varphi}(\mathbf{k})=\mathrm{i} C_{B} B_{0} \frac{\tilde{D}\left(k_{x}, k_{y}, 0\right)}{k_{\perp}^{2}}(\hat{\mathbf{m}} \times \mathbf{k})_{z} ; \quad \tilde{D}(\mathbf{k})=\frac{4 \pi R^{2}}{k} j_{1}(k R) .
$$

Two tilt series were computed, with 36 increments over a $180^{\circ}$ range, resulting in $5^{\circ}$ tilt steps. Each phase map was computed on a grid of $256 \times 256$ nodes. Since the phase is a relatively smoothly varying function, the phases were then interpolated to $2.5^{\circ}$ increments. Filtered back projection was used to compute the magnetic induction components from the tilt series on a $3 \mathrm{D}$ grid with $256^{3}$ nodes. The filter kernel was taken to be the derivative of the standard filtered back projection filter, as suggested in [1]. Fig. 1 shows the input magnetic induction profiles (solid lines) and the reconstructed magnetic induction (symbols) along two lines: $(x, 10,20)$ going through the sphere, and $(-10, y, 40)$ passing next to the sphere. The overall agreement between the two profiles is rather good, indicating that, at least under ideal numerical conditions, a 3D reconstruction of the magnetic induction in and around a particle is feasible.

\section{References}

1. K.B. Winters and D. Rouseff, IEEE Trans. Ultras., Ferroel. and Freq. Contr., 40 (1993) 26

2. M. Beleggia and Y. Zhu, Phil. Mag. B, 83 (2003), 1143.

3. This work was supported by the U.S. Department of Energy, Basic Energy Sciences under contract numbers DE-FG02-01ER45893 and DE-AC02-98CH10886.
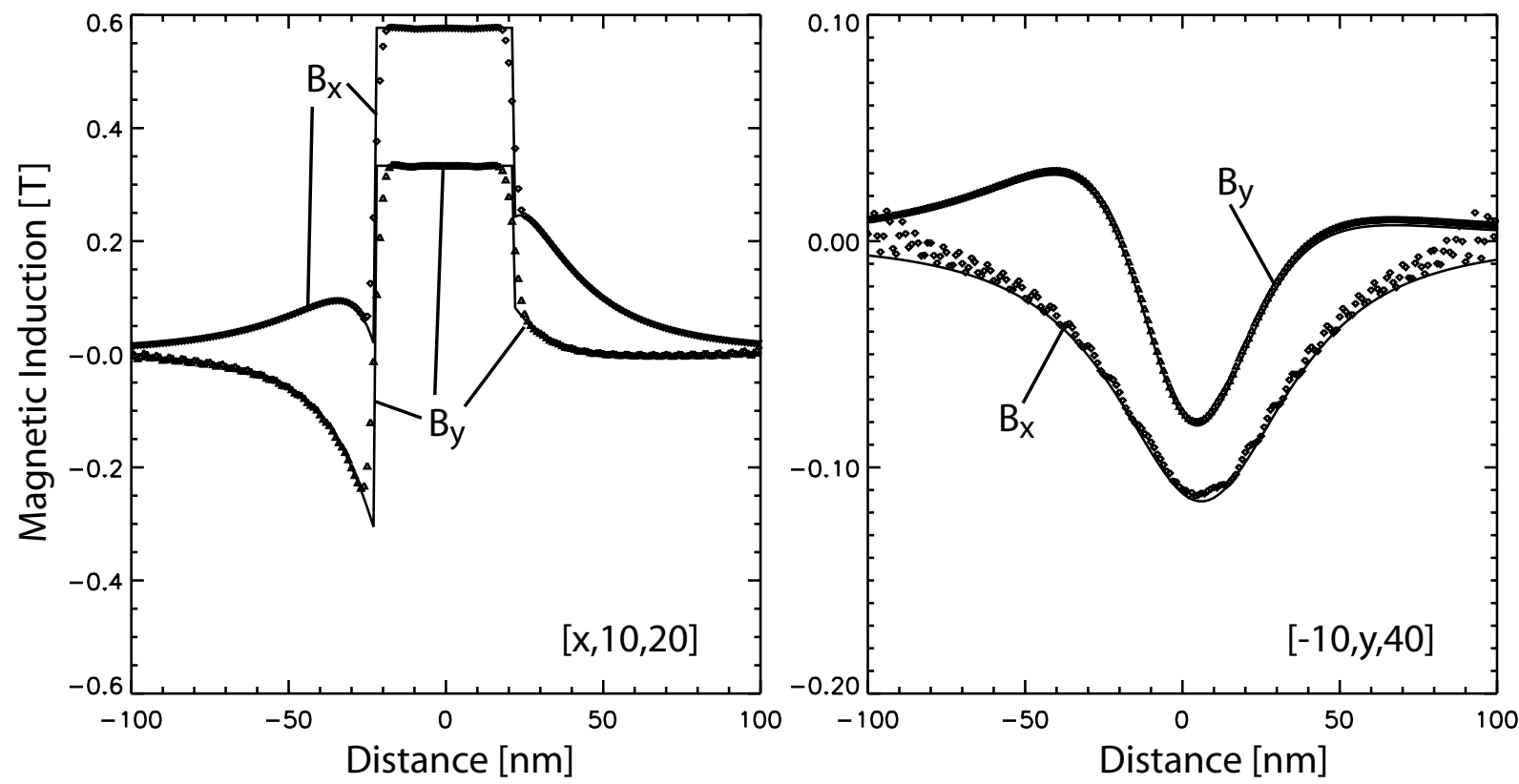

Fig. 1. Comparison between theoretical (solid line) and reconstructed (symbols) magnetic induction profiles along two straight lines through (left) and next to the sphere (right). 